\title{
SIMULATIONS OF AN ACCELERATION SCHEME FOR PRODUCING HIGH INTENSITY AND LOW EMITTANCE ANTIPROTON BEAM FOR FERMILAB COLLIDER OPERATION*
}

\author{
V. Wu, C. M. Bhat, J. A. MacLachlan, FNAL, Batavia, IL 60510, U.S.A.
}

\begin{abstract}
During Fermilab collider operation, the Main Injector (MI) provides high intensity and low emittance proton and antiproton beams for the Tevatron. The present coalescing scheme for antiprotons in the Main Injector yields about a factor of two increase in the longitudinal emittance and a factor of $5 \%$ to $20 \%$ decrease in intensity before injection to the Tevatron. In order to maximize the integrated luminosity delivered to the collider experiments, it is important to minimize the emittance growth and maximize the intensity of the MI beam. To this end, a new scheme using a combination of $2.5 \mathrm{MHz}$ and $53 \mathrm{MHz}$ accelerations has been developed and tested. This paper describes the full simulation of the new acceleration scheme, taking account of space charge, $2.5 \mathrm{MHz}$ and $53 \mathrm{MHz}$ beam loading, and the effect of residual $53 \mathrm{MHz}$ rf voltage during $2.5 \mathrm{MHz}$ acceleration and $\mathrm{rf}$ manipulations. The simulations show the longitudinal emittance growth at the $10 \%$ level with no beam loss. The experimental test of the new scheme is reported in another PAC05 paper.
\end{abstract}

\section{INTRODUCTION}

The Fermilab Tevatron is the highest energy proton and antiproton collider in the world, dedicated to the research on the fundamental constituents of matter. The primary goal of the Fermilab Run II upgrade plan $[1,2]$ is to maximize the integrated luminosity delivered to the Tevatron collider experiments at CDF and D0. To achieve high luminosity, it is essential to preserve the beam emittance and intensity through the accelerator chain. In this effort, beam acceleration and related rf manipulations in the Main Injector play prime role.

Currently, high intensity antiproton bunches in the Main Injector are produced by a multi-bunch coalescing process of $53 \mathrm{MHz}$ bunches at $150 \mathrm{GeV}$ [3]. Four consecutive $2.5 \mathrm{MHz}$ antiproton bunches are injected into the Main Injector from either the Accumulator or the Recycler. Prior to acceleration, each antiproton bunch is adiabatically shrunk and then rebunched into five to nine $53 \mathrm{MHz}$ bunches. The $53 \mathrm{MHz}$ bunches are accelerated from 8 to $150 \mathrm{GeV}$. At $150 \mathrm{GeV}$, each group of antiproton bunches is coalesced into one $53 \mathrm{MHz}$ bunch and then injected into the Tevatron. The coalescing process alone is found to introduce about a factor of two longitudinal emittance growth. The amount of beam loss is in the range of 5 to 20 percent depending on the initial bunch

\footnotetext{
*Operated by Universities Research Association, Inc. for the U.S. Department of Energy under contract DE-AC02-76CH03000.

"vincentw@fnal.gov
}

emittance and intensity. This longitudinal emittance growth can be reduced to the $10 \%$ level with no beam loss using a combination of $2.5 \mathrm{MHz}$ and $53 \mathrm{MHz}$ accelerations $[1,4-6]$ in the Main Injector.

\section{ANTIPROTON ACCELERATION USING 2.5 MHZ (H=28) AND 53 MHZ (H=588) RF SYSTEMS}

\section{Acceleration Scheme}

The antiproton transfers to the Main Injector are in $2.5 \mathrm{MHz}$ buckets and the longitudinal emittance is in the range of 0.8 (from Accumulator) to $3 \mathrm{eVs}$ (Recycler). Hence, it is natural to begin acceleration in $2.5 \mathrm{MHz}$ buckets. This eliminates the rf manipulations in $53 \mathrm{MHz}$ prior to the acceleration and the associated emittance growth. Currently, the maximum $\mathrm{dP} / \mathrm{dt}$ for $2.5 \mathrm{MHz}$ acceleration in the Main Injector is limited by the available rf voltage of $75 \mathrm{kV}$ for the $2.5 \mathrm{MHz}$ rf system. Therefore, we accelerate the beam from $8 \mathrm{GeV}$ to $150 \mathrm{GeV}$ in two steps. From 8 to $27 \mathrm{GeV}$, four consecutive $2.5 \mathrm{MHz}$ bunches are accelerated through transition (at about $20.4 \mathrm{GeV}$ ) using the $2.5 \mathrm{MHz} \mathrm{rf}$ system and a slow acceleration ramp with a maximum $\mathrm{dP} / \mathrm{dt}$ of $3.2(\mathrm{GeV} / \mathrm{c}) / \mathrm{s}$. For a $3 \mathrm{eVs}$ bunch (Recycler beam), the $\Delta \mathrm{P} / \mathrm{P}$ is about $\pm 0.45 \%$, which is about $40 \%$ smaller than the momentum acceptance of the MI at transition energy (about $\pm 0.7 \%$ ). This allows us to accelerate bunches with longitudinal emittance up to about $4 \mathrm{eVs}$ without any beam loss through transition. From 27 to $150 \mathrm{GeV}$, the beam is accelerated with the $53 \mathrm{MHz}$ rf system.

Beam harmonic transfers between the $2.5 \mathrm{MHz}$ and the $53 \mathrm{MHz}$ buckets at constant energy $27 \mathrm{GeV}$ are carried out with two one quarter synchrotron period rotations by the $2.5 \mathrm{MHz}$ rf system. The first rotation is done at low voltage $(\mathrm{a}$ few $\mathrm{kV})$ to reduce the bunch height to an acceptable level (a few $\mathrm{MeV}$ ). Then a fast rotation at high voltage $(60 \mathrm{kV})$ follows. When the bunches are at minimum bunch widths (less than $18.9 \mathrm{nsec}$, the width of $53 \mathrm{MHz}$ bucket), they are captured in matched $53 \mathrm{MHz}$ buckets. In addition to the $2.5 \mathrm{MHz}$ voltages, second harmonic $(\mathrm{h}=56)$ voltages are added to linearize the quarter synchrotron period rotations. For the first and the second rotations, about $10 \%$ and $16 \%$ of the second harmonic are added, respectively. This reduces emittance dilution due to non-linearity in the rf wave form for large emittance beam. 


\section{Multiparticle Beam Dynamics Simulations}

Longitudinal beam dynamics simulations are performed with a macro-particle Monte Carlo tracking code ESME [7]. The simulations are performed for the following cases: (1) space charge effect and broadband impedance, (2) with and without beam loading compensation on the 2.5 MHz rf system and (3) with and without beam loading compensation on the $53 \mathrm{MHz}$ rf system. Simulations were also carried out to understand the effect of the $53 \mathrm{MHz}$ rf voltage on the beam during $2.5 \mathrm{MHz}$ acceleration and during the rf manipulations at $27 \mathrm{GeV}$.

The acceleration scheme is first simulated without the effects of space charge, beam loading and $53 \mathrm{MHz}$ rf voltage to provide a reference for minimum emittance growth for the ideal situation. Figure 1 shows some phase

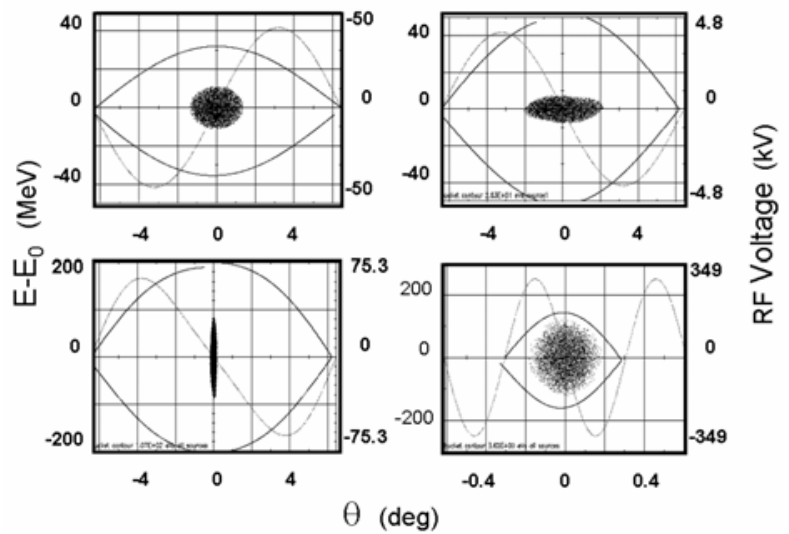

Figure 1: Phase space $(\Delta \mathrm{E}, \theta)$ distributions from 8 to $150 \mathrm{GeV}$. First plot is $2.5 \mathrm{MHz}$ bunch at injection. Second plot is the end of first rotation at $27 \mathrm{GeV}$ at minimum bunch height. Third plot is the end of second rotation at $27 \mathrm{GeV}$ at minimum bunch width. Fourth plot is the $53 \mathrm{MHz}$ bunch at $150 \mathrm{GeV}$.

space plots of the simulation. Transition crossing is performed simply by jumping the acceleration phase from the positive slope to the negative slope of the sinusoidal rf wave form. There is about 3\% emittance growth for a $1.5 \mathrm{eVs}$ beam bunch crossing transition. During the harmonic beam transfer at $27 \mathrm{GeV}$, the rf manipulations give about $5 \%$ emittance growth. The overall acceleration efficiency is $100 \%$.

The effects of space charge and beam pipe coupling impedance on the beam are simulated with a single bunch. The beam pipe is assumed to be a broadband resonator. The estimated Main Injector longitudinal impedance per harmonic $\mathrm{Z}_{11} / \mathrm{n}=1.6 \Omega$ [8] is used. Generally, space charge is more problematic on low longitudinal emittance beam with high intensity. Therefore, simulation is performed for the case of $0.8 \mathrm{eVs}$ initial emittance and $170 \times 10^{9}$ bunch intensity. Simulation shows that there is less than $10 \%$ overall emittance growth without beam loss. Further simulations show that there is no significant (less than 20\%) emittance growth within a factor of three of $Z_{11} / n=1.6 \Omega$.
For the simulations with $2.5 \mathrm{MHz}$ beam loading, four bunches and one cavity are modeled in ESME. The Main Injector has five $2.5 \mathrm{MHz}$ cavities. The shunt impedance of the cavity is increased by a factor of five to simulate the total beam loading. Simulations predict a significant amount of phase shift and filamentation to the bunches, which resulted in a considerable amount of emittance dilution as well as beam loss. Figure 2 shows the beam

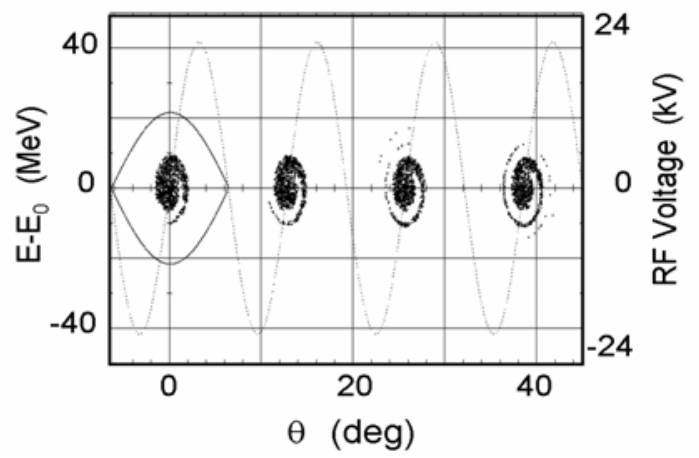

Figure 2: Phase space $(\Delta \mathrm{E}, \theta)$ distribution showing the effect of $2.5 \mathrm{MHz}$ beam loading at injection.

loading effect. Undesirable effects on the bunches are first seen at $8 \mathrm{GeV}$ when the $2.5 \mathrm{MHz}$ voltage is small and comparable to the beam loading voltage. At the constant energy $27 \mathrm{GeV}$, the accumulated phase shift due to beam loading is rather large, e.g., more than 45 degrees. This creates severe bucket mismatch when the beam is transferred to the $53 \mathrm{MHz}$ buckets. The maximum $2.5 \mathrm{MHz}$ beam loading voltage is about $2.1 \mathrm{kV}$. In conclusion, $2.5 \mathrm{MHz}$ beam loading compensation (BLC) is needed.

The physical effects of the feed-back BLC and the feedforward BLC are to lower the quality factor (Q) of the cavity and to reduce the beam loading voltage, respectively. Therefore, the amount of required beam loading compensations can be estimated by reducing the cavity $\mathrm{Q}$ and the charge of the beam in the simulations. Simulations assuming a factor of 5 reduction in the cavity $\mathrm{Q}$ and a factor of 10 reduction in the beam charge are performed. Three cases of initial emittance, i.e., 0.8, 1.5 and $2.2 \mathrm{eVs}$ with nominal bunch intensity $170 \times 10^{9}$ are studied. For the cases of 1.5 and $2.2 \mathrm{eVs}$, beam loadings are not problematic. The case of $0.8 \mathrm{eVs}$ shows that the emittance growth of the last bunch is about seven times larger than that of the first bunch. This indicates that more beam loading compensation is needed for this case. To reduce the emittance growth to the $10 \%$ level, a factor of 30 reduction in the beam loading voltage from the feedforward BLC is needed.

The Main Injector has eighteen $53 \mathrm{MHz}$ cavities with a shunt impedance of $520 \mathrm{k} \Omega$ and a quality factor of 5000 . The beam loading effects of these cavities on the beam can't be ignored. During the $2.5 \mathrm{MHz}$ acceleration, $53 \mathrm{MHz}$ beam loading can potentially affect the beam during transition crossing because the $2.5 \mathrm{MHz}$ bunches at transition have time structures similar to those of the $53 \mathrm{MHz}$. Also, at $27 \mathrm{GeV}, 53 \mathrm{MHz}$ beam loading may 
affect the bunch rotations and the harmonic transfers to the $53 \mathrm{MHz}$ buckets.

The effects of $53 \mathrm{MHz}$ beam loading on the beam are simulated with four bunches and one cavity. The shunt impedance of the cavity is multiplied by a factor of 18 to simulate beam loadings of the 18 cavities. Simulation with bunch intensity of $60 \times 10^{9}$ and initial emittance of $1.5 \mathrm{eVs}$ indicates that $53 \mathrm{MHz}$ beam loading has adverse effects on the beam. The beam loading voltage causes fragmentation of the bunches when crossing transition. See figure 3. This resulted in large emittance dilution and beam loss when the bunches are transferred to $53 \mathrm{MHz}$ buckets at $27 \mathrm{GeV}$. During $2.5 \mathrm{MHz}$ acceleration, the beam loading voltage reaches a maximum of $12 \mathrm{kV}$ at transition. The peak beam loading voltage during $53 \mathrm{MHz}$ acceleration is $35 \mathrm{kV}$. In conclusion, $53 \mathrm{MHz}$ beam loading compensation is required.

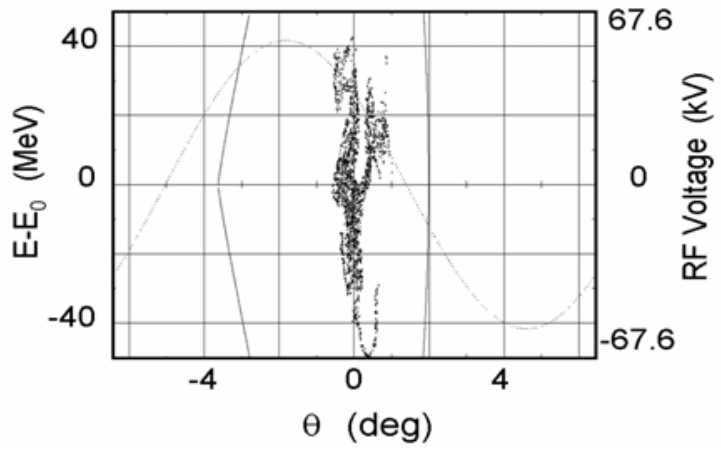

Figure 3: Simulation with $53 \mathrm{MHz}$ beam loading during $2.5 \mathrm{MHz}$ acceleration. Phase space $(\Delta \mathrm{E}, \theta)$ plot shows the first bunch after transition.

Simulations with $53 \mathrm{MHz}$ beam loading assuming a factor of 5 reduction in the cavity $\mathrm{Q}$ and a factor of 10 reduction in the beam charge are performed. Two cases of bunch intensity, $60 \times 10^{9}$ and $170 \times 10^{9}$, with initial emittance of $1.5 \mathrm{eVs}$ are studied. Simulation shows that beam loading in the case of bunch intensity $60 \times 10^{9}$ is insignificant. For the case of $170 \times 10^{9}$, the emittance growth of the last bunch is three times larger than that of the first bunch. To reduce the emittance growth of the last bunch to the $10 \%$ level, the feedforward BLC needs to provide a factor of 30 reduction in the beam loading voltage.

During the $2.5 \mathrm{MHz}$ acceleration from 8 to $27 \mathrm{GeV}$, it is undesirable to short the Main Injector $53 \mathrm{MHz} \mathrm{rf}$ cavities. The $53 \mathrm{MHz}$ rf systems need to be in tune with the MI magnet ramp so that at $27 \mathrm{GeV}$ they can be turned on smoothly when the $2.5 \mathrm{MHz}$ bunches are transferred to $53 \mathrm{MHz}$ buckets. However, keeping the $53 \mathrm{MHz}$ rf systems at low voltage during $2.5 \mathrm{MHz}$ acceleration may cause transition crossing problem and filamentation to the $2.5 \mathrm{MHz}$ bunches because of the $53 \mathrm{MHz}$ modulation to the $2.5 \mathrm{MHz}$ rf wave form. Therefore, the effect of the $53 \mathrm{MHz}$ voltage on $2.5 \mathrm{MHz}$ acceleration needs to be studied.

To understand this effect, a series of single bunch simulations are performed with various amount of constant $53 \mathrm{MHz}$ rf wave superimposed on the $2.5 \mathrm{MHz}$ accelerating $\mathrm{rf}$ wave form. Simulations show that the bunch behaves fairly well before transition. However, with high enough $53 \mathrm{MHz}$ voltage, filamentation of the bunch occurs after transition crossing. The higher momentum particles are separated from the core and form two spiral arms. During the first rotation (with $4 \mathrm{kV}$ of $2.5 \mathrm{MHz}$ voltage) at $27 \mathrm{GeV}$, the bunch develops $53 \mathrm{MHz}$ structure as expected. Subsequently, this creates significant amounts of emittance growth and beam loss. The $53 \mathrm{MHz}$ voltage during $2.5 \mathrm{MHz}$ acceleration needs to be reduced to $500 \mathrm{~V}$ or less in order to have emittance dilution at the $10 \%$ level.

\section{CONCLUSION}

We need $2.5 \mathrm{MHz}$ and $53 \mathrm{MHz}$ feed-back and feedforward beam loading compensations in order to keep emittance growth less than $20 \%$ with no beam loss. The minimal requirements for the $2.5 \mathrm{MHz}$ and the $53 \mathrm{MHz}$ beam loading compensations are a factor of 5 reduction in the cavity Q from the feed-back BLC and a factor of 10 reduction in the beam loading voltage from the feedforward BLC. For small emittance (e.g., $0.8 \mathrm{eVs}$ ) and large bunch intensity (e.g., $170 \times 10^{9}$ ) beam, the requirements for the $2.5 \mathrm{MHz}$ and the $53 \mathrm{MHz}$ feedforward BLC are a factor of 30 reduction in the beam loading voltage. The limit on the $53 \mathrm{MHz}$ rf voltage during $2.5 \mathrm{MHz}$ acceleration and bunch rotations is $500 \mathrm{~V}$ or less. The effects of the space charge and the beam pipe coupling impedance on the beam are insignificant.

\section{REFERENCES}

[1] G. P. Jackson, Fermilab Recycler Ring Technical Design Report, FERMILAB-TM-1991, November 1996.

[2] "Fermilab Collider Run II: Accelerator Status and Upgrades", P. C. Bhat and W. J. Spalding, to be published in the Proceedings of HCP2004, Michigan; "The Run II Luminosity Upgrade at the Fermilab Tevatron - Project Plan and Resources-Loaded Schedule", Internal Accelerator Division Document, June 2003.

[3] J. E. Griffin et al, IEEE, Trans. Nucl., Sci., NS-30, No. 4, (1983) 2627; C. M. Bhat et al, this proceedings.

[4] I. Kourbanis and D. W. Wildman, PAC1999, New York (1999), page 2840.

[5] J. A. MacLachlan, FERMILAB-TM-2177, July 2002.

[6] C. M. Bhat and J. E. Dey, "Pbar Acceleration in MI using 2.5 MHz Coalescing RF system - Tune-up Study Scheme with Proton Beam and Operational Implementation", MI Note 272, February 2001.

[7] J. A. MacLachlan, User's Guide to ESME 2002, Fermilab, November 2002.

[8] W. Chou, PAC1997, Vancouver, Canada (1997), page 991. 